\title{
Association of Gleason score with PSA Values and Serum Testosterone Levels Measured Prior To Prostate Biopsy
}

\author{
Muhammed Cihan Temel ${ }^{1}$, Caner Ediz', Serkan Akan², Eymen Ozer ${ }^{2}$ and Omer Yilmaz ${ }^{2}$ \\ ${ }^{1}$ Department of Urology, Nevsehir State Hospital, Nevsehir, Turkey \\ ${ }^{2}$ Department of Urology, Sultan Abdulhamid Han Education and Research Hospital, Istanbul, Turkey
}

\begin{abstract}
Objective: To analyse the association of prostate cancer (PCa) Gleason score evaluated upon prostate biopsy with clinical PSA values, total and free testosterone (TT and FT, respectively) levels measured prior to biopsy.

Study Design: A descriptive study.

Place and Duration of Study: Department of Urology, Sultan Abdulhamid Han Education and Research Hospital, from July to December 2019.

Methodology: A total of 85 patients were included and classified into non-PCa (group 1) and PCa (group 2) groups according the results of prostate biopsy pathology. Age, digital rectal examination (DRE) findings; prostate volume (PV); free/total prostate specific antigen (PSA) ratio ( $f / t P S A)$; PSA density (PSA-D) and total PSA (tPSA), free PSA (fPSA), $T$ and FT levels of the two groups were evaluated. Associations of the ISUP grade of patients in group 2 with age, DRE findings for PCa; PV; PSA-D; f/tPSA and tPSA, fPSA, TT and FT levels were analysed.

Results: Mean patient age was 63.00 (57.50-70.00) years. Mean age, significant DRE findings for PCa, tPSA levels and PSA-D were significantly higher in group $2(p<0.05)$, whereas PV was significantly higher in group $1(p<0.05)$. The ISUP grade of patients in group 2 was significantly and positively correlated with age and tPSA levels $(p<0.05)$. ISUP grade was significantly and positively correlated with significant DRE findings for PCa $(p<0.05)$. However, ISUP grade was negatively correlated with PV and f/tPSA ( $p$ $<0.05)$. No significant difference was observed between the two groups in terms of $\pi$ and FT levels $(p>0.05)$.

Conclusion: TT and FT levels evaluated before prostate biopsy did not provide any additional benefit in predicting Gleason score grade before biopsy.
\end{abstract}

Key Words: Prostate biopsy, prostate cancer, ISUP grade, Gleason score, total testosterone, free testosterone

How to cite this article: Temel MC, Ediz C, Akan S, Ozer E, Yilmaz O. Association of Gleason score with PSA Values and Serum Testosterone Levels Measured Prior To Prostate Biopsy. J Coll Physicians Surg Pak 2020; 30(04):399-402. DOI: https://doi.org/10.29271/jcpsp.2020.04.399.

\section{INTRODUCTION}

Prostate cancer ( $\mathrm{PCa}$ ) is the most common non-cutaneous cancer in men in the United States. ${ }^{1}$ It is responsible for $10 \%$ of all cancerrelated deaths and is the second most common malignancy after lung cancer. Approximately $75 \%$ of diagnosed PCa cases are patients aged $\geq 65$ years, and the disease incidence increases with age. ${ }^{2}$ Androgens and androgen receptors are important for prostate growth and development as well as PCa development. ${ }^{3}$ Porcaro et al. reported a positive correlation between basal total testosterone (TT) levels and ISUP grade reported at diagnosis. ${ }^{4}$ In a collaborative analysis of 20 prospective studies, men with low circulating free testosterone (FT) levels were likely to be at a lower risk of overall PCa. ${ }^{5}$

Correspondence to: Caner Ediz, Department of Urology, Sultan Abdulhamid Han Education and Research Hospital, Tibbiye Street, Selimiye Neighborhood, Uskudar/Istanbul, Postal Code 34668, Turkey

E-mail:drcanerediz@gmail.com

Received: March 14, 2020; Revised: April 30, 2020;

Accepted: May 08, 2020

DOI: https://doi.org/10.29271/jcpsp.2020.04.399
Moreover, some parameters such as patient age, digital rectal examination (DRE) finding, total prostate specific antigen (tPSA) level, PSA density (PSA-D) and free/total PSA (f/tPSA) ratio are associated with PCa risk and Gleason grade. ${ }^{6-10}$ Thus, Gleason score (GS) for PCa is affected by various parameters.

The association between GS and androgen levels has only been retrospectively evaluated in the past.

The aim of this study was to prospectively analyse the association of ISUP grade with clinical PSA values and testosterone levels evaluated prior to prostate biopsy and to define the parameters that worsen ISUPgrade, ifany.

\section{METHODOLOGY}

Patients who were admitted to the Urology Outpatient Clinic of Sultan Abdulhamid Han Education and Research Hospital between July 2019 and December 2019 with complaints of lower urinary tract symptoms and for whom a prostate biopsy decision was made due to significant DRE findings for PCa and/or TPSA elevation based on physical examination and other evaluations were included. This prospective descriptive study was approved by the local Ethics Committee and conducted according to the principles of World Medical Association Declaration of Helsinki 'Ethical Principles for Medical Research Involving Human Subjects. 
Patients aged $\geq 50$ years who underwent prostate biopsy due to the presence of significant DRE findings for PCa and/or TPSA elevation were included in the study. Exclusion criteria were non-consent to participate in the study; age $<50$ years; urinary system infection that may affect patient's hormonal parameters (tPSA, fPSA, TT and FT levels; f/tPSA ratio and PSA-D); recent invasive urinary system intervention and presence of any additional pathology such as testicular/pituitary/hypothalamic defect, diabetes, obesity $\left(\mathrm{BMI}>30 \mathrm{Kg} / \mathrm{m}^{2}\right)$, severe cardiovascular disease or metabolic syndrome. A total of 85 patients who met these criteria were included in the study.

Table I: Comparison of age; PV; f/tPSA; PSA-D and tPSA, fPSA, TT and FT levels between groups 1 and 2 .

\begin{tabular}{|c|c|c|c|c|}
\hline Parameter & Pathology & $\mathbf{n}$ & $\begin{array}{c}\text { Median (interquartile } \\
\text { range) }\end{array}$ & p-value \\
\hline \multirow{2}{*}{ Age (years) } & Group 1 & 52 & $59.50(56.50-68.00)$ & \multirow{2}{*}{$0.001 *$} \\
\hline & Group 2 & 33 & $65.00(61.00-73.00)$ & \\
\hline \multirow{2}{*}{ Prostate volume (cc) } & Group 1 & 52 & $48.50(40.00-62.00)$ & \multirow{2}{*}{$0.002^{*}$} \\
\hline & Group 2 & 33 & $36.00(30-48.00)$ & \\
\hline \multirow{2}{*}{ Total PSA (ng/mL) } & Group 1 & 52 & $5.16(3.94-7.14)$ & \multirow{2}{*}{$0.022^{*}$} \\
\hline & Group 2 & 33 & $6.72(5.09-11.78)$ & \\
\hline \multirow{2}{*}{ Free PSA (ng/mL) } & Group 1 & 52 & $0.98(0.68-1.73)$ & \multirow{2}{*}{0.291} \\
\hline & Group 2 & 33 & $1.19(0.76-1.73)$ & \\
\hline \multirow{2}{*}{ Free/total PSA ratio } & Group 1 & 52 & $0.19(0.17-0.25)$ & \multirow{2}{*}{0.058} \\
\hline & Group 2 & 33 & $0.17(0.12-0.23)$ & \\
\hline \multirow{2}{*}{ PSA density (ng/mL/cc) } & Group 1 & 52 & $0.10(0.09-0.15)$ & \multirow{2}{*}{0.001} \\
\hline & Group 2 & 33 & $0.21(0.15-0.34)$ & \\
\hline \multirow{2}{*}{ Total testosterone (ng/dL) } & Group 1 & 52 & 456.39 (373.82-535.85) & \multirow{2}{*}{0.223} \\
\hline & Group 2 & 33 & $561.60(403.97-612.00)$ & \\
\hline \multirow{2}{*}{ Free testosterone (ng/dL) } & Group 1 & 52 & $9.63(6.92-12.82)$ & \multirow{2}{*}{0.256} \\
\hline & Group 2 & 33 & $8.97(6.75-10.30)$ & \\
\hline \multicolumn{5}{|c|}{$\begin{array}{l}\text { Values are presented as median (interquartile range). } * p<0.05 \text {. } \\
\text { Median TT levels in groups } 1 \text { and } 2 \text { were } 456.39(373.82-535.85) \text { and } 561.60(403.97-612.00) \\
\text { ng/dL, respectively. Median FT levels in groups } 1 \text { and } 2 \text { were } 9.63(6.92-12.82) \text { and } 8.97 \\
(6.75-10.30) \text { ng/dL, respectively. Comparative analysis between the two groups revealed no } \\
\text { difference in terms of TT and FT levels }(p>0.05 \text {, Table I). Results of comparison of age; PV; } \\
\text { f/tPSA; PSA-D and tPSA, fPSA, TT and FT levels are shown in Table I. Linear regression } \\
\text { analysis with ISUP grade as the dependent variables and age; PV; f/tPSA; PSA-D and tPSA, } \\
\text { fPSA TT and FT levels as the independent variables revealed a significant positive correlation } \\
\text { of ISUP grade with age and tPSA levels ( } p=0.003 \text { and } p<0.001 \text {, receptively; Table II) and a } \\
\text { significant negative correlation of ISUP grade with PV and f/tPSA levels }(p<0.001 \text { and } p= \\
0.048, \text { receptively; Table II). }\end{array}$} \\
\hline
\end{tabular}

Table II: Linear regression analysis with ISUP grade as the dependent variables and age; PV; f/tPSA; PSA-D and tPSA, fPSA TT and FT levels as the independent variables.

\begin{tabular}{|c|c|c|c|c|}
\hline \multirow[t]{2}{*}{ Parameter } & \multirow{2}{*}{$\begin{array}{c}\text { Median } \\
\text { (interquartile range) }\end{array}$} & \multirow[t]{2}{*}{$\beta$ value } & \multirow[t]{2}{*}{ p-value } & \begin{tabular}{|c|}
$95 \%$ confidence \\
interval for $\beta$
\end{tabular} \\
\hline & & & & Lower to upper \\
\hline Age (years) & $63(57.50-70.00)$ & 0.196 & $0.003^{*}$ & -0.01 to 0.04 \\
\hline Prostate volume (cc) & $45.00(32.5-59.35)$ & -0.342 & $<0.001 *$ & -0.02 to 0.01 \\
\hline Total PSA (ng/mL) & $5.59(4.00-8.53)$ & 1.39 & $<0.001 *$ & 0.04 to 0.11 \\
\hline Free PSA (ng/mL) & $1.06(0.72-1.73)$ & -0.37 & 0.19 & -0.19 to 0.04 \\
\hline Free / total PSA ratio & $0.18(0.15-0.25)$ & -0.15 & $0.048^{*}$ & -3.80 to 0.02 \\
\hline PSA density (ng/mL/cc) & $0.12(0.09-0.22)$ & -0.27 & 0.15 & -1.58 to 0.25 \\
\hline Total testosterone (ng/dL) & 486.67 (383.73-593.59) & 0.09 & 0.18 & 0.00 to 0.00 \\
\hline Free testosterone (ng/dL) & $9.44(6.86-11.41)$ & -0.07 & 0.25 & -0.05 to 0.01 \\
\hline $\begin{array}{l}\text { Digital rectal examination } \\
\text { findings }\end{array}$ & Odds Ratio: 2.63 & 0.97 & $0.003^{*}$ & 1.40 to 4.92 \\
\hline
\end{tabular}

Age, significant DRE findings for PCa (presence of nodules and diffuse stiffness, amongst others); prostate volume (PV) measured by transrectal ultrasonography; tPSA, fPSA, TT and FT levels; f/tPSA ratio and PSA-D were evaluated, and the results were prospectively entered into a data collection system. Blood samples required to test these parameters were obtained between 8 and 10 am just before prostate biopsy. Thus, biopsy was not affected by DRE. No restriction was imposed on patients' sexual activities to evaluate hormonal levels. The patients were classified into non-PCa (group 1) and PCa (group 2) groups according to the results of prostate biopsy pathology. Both groups were statistically compared in terms of the aforementioned parameters. The correlations of ISUP grade with the measured parameters were evaluated by linear regression analysis, with ISUP grade as the dependent variable and age; PV; f/tPSA; PSA-D and tPSA, fPSA, TT and FT levels as independent variables. In addition, binary logistic regression analysis was performed to investigate the association between DRE findings and ISUP grade, with the presence/absence of significant DRE findings for PCa was used as the dependent variables and ISUP grade as the independent variable. GS was classified according to the current 2014 ISUP grading system.

One day before prostate biopsy, oral administration of $500 \mathrm{mg}$ levofloxacin and $400 \mathrm{mg}$ etodolac was initiated and continued for five days. On the day of biopsy, rectal enema $(250 \mathrm{~mL})$ was performed prior to prostate biopsy. The procedure was performed with the patient in the left lateral position with flexed thighs. The procedure was performed under ultrasound guidance using a 7.5$\mathrm{MHz}$ biplanar probe. Biopsy was performed on an outpatient basis in a room equipped with all materials necessary for emergency intervention. Sedation and anaesthesia were not achieved. Ten minutes before the procedure, periprostatic nerve blockade was performed using perianal intrarectal lidocaine gel. Injections were delivered at the angle between the seminal vesicle and prostate on each side using $5 \mathrm{cc}$ of $2 \%$ lidocaine. The 12 quadrant prostate biopsies were performed by multiple experienced urologists.

Statistical analysis was performed using SPSS for Windows 22.00. Shapiro-Wilk test was used to assess normal data distribution. Qualitative variables were expressed as frequency and percentage, and Chi-square test was applied. Quantitative variables were expressed as median (interquartile range). All variables were non-normally distributed. Therefore, Mann-Whitney's $U$ test was used to evaluate all non-normally distributed variables. Pearson correlation, linear logistic regression and binary logistic regression analyses were performed to assess the correlations amongst variables. Pearson correlation analysis was applied as a preliminary study for linear regression analysis (data not included here), and meaningful results were obtained with linear regression analysis. Binary logistic regression analysis was used to assess the correlation between the presence/absence of significant DRE findings for PCa and ISUP grade. Simple linear regression analysis was to assess the correlations of ISUP grade with age; PV; f/tPSA; PSA-D and tPSA, fPSA, TT and FT levels. A $p<0.05$ was considered statistically significant in all analyses.

\section{RESULTS}

Mean (range) patient age was 63.00 (57.50-70.00) years. Median PV was 45.00 (32.50-59.35) cc. Median tPSA and fPSA levels were $5.59(4-8.53)$ and $1.06(0.72-1.74) \mathrm{ng} / \mathrm{mL}$, respectively. Median f/tPSA ratio was $0.18(0.15-0.25)$, and median PSA-D was 0.12 (0.09-0.22) ng/mL/cc. Median TT and FT levels were 486.67 (383.73-593.59) and 9.44 (6.86-11.41) ng/dL, respectively.

Furthermore, 33 patients (38.82\%) showed significant DRE 
findings for PCa and 52 (61.18\%) did not. According to the results of prostate biopsy pathology, 52 patients $(61.18 \%)$ were included in the non-PCa (group 1) and 33 (38.82\%) in the PCa (group 2) group.

Median patient age was 59.50 (56.50-68.00) years in group 1 and $65.00(61.00-73.00)$ years in group 2. Moreover, $19(22.4 \%)$ and $14(16.5 \%)$ patients showed significant DRE findings for PCa in groups 2 and 1 , respectively. The rate of detecting significant DRE findings for PCa was significantly higher in group 2 than in group 1 $(p=0.005)$. Binary logistic regression analysis was performed with the presence/absence of significant DRE findings for PCa as the dependent variable and ISUP grade as the independent variable, which revealed a significant positive correlation between ISUP grade and the presence of significant DRE findings for PCa $(p=0.003)$.

Median PV was 48.50 (40.00-62.00) cc in group 1 and 36.00 (30-48.00) cc in group 2. PV was significantly higher in group 1 ( $p$ $=0.002)$. Median tPSA level was 5.16 (3.94-7.14) ng/mL in group 1 and $6.72(5.09-11.78) \mathrm{ng} / \mathrm{mL}$ in group 2. Median PSA-D was 0.10 $(0.09-0.15) \mathrm{ng} / \mathrm{mL} / \mathrm{cc}$ in group 1 and $0.21(0.15-0.34) \mathrm{ng} / \mathrm{mL} / \mathrm{cc}$ in group 2. Age, tPSA level and PSA-D were significantly higher in group 2 ( $p=0.001, p=0.022$ and $p=0.001$, respectively). No statistically significant difference was observed in terms of fPSA levels and f/tPSA between the two groups $(p=0.058$, Table I).

\section{DISCUSSION}

PCa is the second most common cancer in men and the fifth most common cause of mortality worldwide. ${ }^{11}$ Prostate development and growth are closely associated with androgenic stimulation. The association between serum $\Pi$ levels and PCa has become increasingly clear in the recent years. In a cohort study conducted in the USA, Muralidhar et al. reported that older men are more likely to have high-grade PCa or show a high risk of developing $\mathrm{PCa}{ }^{8}$ Meanwhile, Alibhai et al. attributed even a slight increase in PCa stage and grade at the time of diagnosis to possible delayed diagnosis in older men rather than advanced age. ${ }^{12}$ In the present study, a significant positive correlation was observed between age and GS. However, considering the results of regression analysis in which the shared effect of all the above-mentioned parameters on GS was investigated, our results are consistent with those of Alibhai etal. ${ }^{12}$

By examining the relationship between PCa and DRE, Carvalhal et al. reported that the positive predictive value of significant DRE findings for PCa was significant in men with low serum tPSA levels; this value was $5 \%, 14 \%$ and $30 \%$ in men with tPSA levels of $0-1.0$, 1.1-2.5 and 2.6-4.0 ng/mL, respectively, and all diagnosed cancers were clinically localised. They also reported that most of these detected cancers were clinically significant and treatable. ${ }^{6}$ In the present study, a significant positive correlation was observed between the presence of significant DRE findings for $\mathrm{PCa}$ and ISUP grade; therefore, our results are consistent with those of Carvalhal etal. ${ }^{6}$

Mir et al. investigated the relationship between PCa and PV and, based on multivariate analysis, reported that PV measured using transrectal ultrasonography was negatively correlated with high grade tumour rate determined by pathological examination following transrectal needle biopsy or radical prostatectomy. ${ }^{13}$ In another study, Caliskan et al. reported that smaller prostates are more likely to compose higher percentage of high-grade prostate cancer, local advanced disease and Gleason upgrading. ${ }^{14}$ Consistent with the results of both studies, we observed a significant negative correlation between PV and ISUP grade in multivariate regression analysis in the present study.

PSA forms represent an important adjunct to DRE for the detection of PCa. ${ }^{15}$ Fang et al. investigated the association between PCa and PSA forms and reported that serum tPSA levels were positively correlated with postoperative GS. ${ }^{16}$ According to the results of multivariate regression analysis in the present study, there was a significant positive correlation between tPSA levels and ISUP grade ( $p<0.05$ ). $f$ /tPSA ratio is specifically used in "grey zone" patients. ${ }^{17}$ In a study by Elabbady and Khedr, men with PCa and low $\mathrm{f} / \mathrm{tPSA}$ ratio were at an increased risk of showing a high GS (7-10) and those with high f/tPSA ratio were more likely to show a low GS. ${ }^{18}$ According to the present data, there was a significant negative correlation between f/tPSA ratio and ISUP grade ( $p<$ $0.05)$, which is consistent with the study of Elabbady and Khedr.

Drobková et al. evaluated androgen presence and PCa and reported that serum TT was not a significant predictive factor for pathological GS and stage. ${ }^{19}$ In the present study, there was no significant difference in TT levels between the non-PCa and $\mathrm{PCa}$ groups based on the results of pathological evaluation. Furthermore, no positive or negative correlation was observed between TT levels and ISUP grade. In another study on such patients, Morote et al. reported that PCa risk and tumour aggressiveness were not associated with serum TT and FT levels. ${ }^{20}$ In the present study, no significant relationship was observed between FT levels and PCa risk or risk of increase in ISUP grade $(p<0.05)$. In addition, there was no statistically significant difference between the two groupsinterms of FTlevels ( $p<0.05)$.

Patients' serum hormonal levels were based solely on circulating levels, which may be a limitation of the present study. Data on patients' comorbidities were collected in this study, and comorbidities that could affect hormone levels were excluded; however, no additional evaluation was performed by an expert endocrinologist. Nonetheless, all patient parameters were measured in the same laboratory at a single centre.

\section{CONCLUSION}

In patients who underwent prostate biopsy, age, DRE findings for PCa and tPSA levels were positively correlated with increased PCa and ISUP grade risks. However, there was a negative correlation of ISUP grade with PV and f/tPSA ratio. In addition, PSA-D was significantly higher in patients with PCa than in those without PCa based on pathology results. No association of $\Pi$ and FT levels with the presence of PCa or increase in ISUP grade was observed. In case of suspicion of $\mathrm{PCa}$, prior consideration and evaluation of parameters such as patient age, DRE findings, tPSA, f/tPSA, PV and PSA-D can guide the decision for biopsy. Evaluation of TT and FT levels prior to prostate biopsy offers no additional clinical benefit.

\section{ETHICAL APPROVAL:}

This prospective descriptive study was approved by the local Ethics Committee of Haydarpaşa Numune Training and Research Hospital (HNEAH-KAEK 2019/143). 


\section{PATIENTS' CONSENT:}

Informed consent is obtained from patients to publish the data concerning this study.

\section{CONFLICT OF INTEREST:}

Authors declared no conflict of interest.

\section{AUTHORS' CONTRIBUTION:}

EC: Contributed to the conception and design of the study.

EC, TMC, OE, AS: Collected the data.

$E C, Y O, A S$ : Drafted and revised the manuscript.

EC, AS, TMC, OE: Participated in preparing tables and performing statistical analysis.

\section{REFERENCES}

1. Siegel RL, Miller KD, Jemal A. Cancer statistics 2017. CA Cancer J Clin 2017; 67:7-30.

2. Rosai J. Male reproductive system, Prostate and seminal vesicles. Ackerman's Surg Pathol 1996; 1221-56.

3. Lonergan PE, Tindall DJ. Androgen receptor signaling in prostate cancer development and progression. J Carcinog 2011; 10:20.

4. Porcaro AB, Tafuri A, Sebben M, Pirozzi M, Processali T, Rizzetto $R$, et al. Positive association between basal total testosterone circulating levels and tumor grade groups at the time of diagnosis of prostate cancer. Urol Int 2019; 103: 400-7.

5. Watts EL, Appleby PN, Perez-Cornago A, Bueno-de-Mesquita $H B$, Chan JM, Chen C, et al. Low free testosterone and prostate cancer risk: A collaborative analysis of 20 prospective studies. Eur Urol 2018; 74:585-94.

6. Carvalhal GF, Smith DS, Mager DE, Ramos C, Catalona WJ. Digital rectal examination for detecting prostate cancer at prostate specific antigen levels of $4 \mathrm{ng} . / \mathrm{ml}$. or less. J Urol 1999; 161:835-9.

7. Karademir I, Shen D, Peng Y, Liao S, Jiang Y, Yousuf A, et al. Prostate volumes derived from MRI and volume-adjusted serum prostate-specific antigen: Correlation with Gleason score of prostate cancer. AJR Am J Roentgenol 2013; 201: 1041-8.

8. Muralidhar V, Ziehr DR, Mahal BA, Chen YW, Nezolosky MD, Viswanathan VB, et al. Association between older age and increasing Gleason score. Clin Genitourin Cancer 2015; 13: 525-30.e1-3.

9. Ngwu P, Achor G, Eziefule V, Orji J, Alozie F. Correlation between prostate specific antigen and prostate biopsy Gleason score. Ann Health Res 2019; 5:243-8.

10. Yazdani M, Baradaran A, Tamadon MR, Khodadadi S, Kabiri M, Koushki AM, et al. Association of serum total PSA level and free-to-total PSA ratio with grade of prostate cancer in biopsy specimens. Immunopathol Persa 2016; 2:e21.

11. Rawla P. Epidemiology of prostate cancer. World J Oncol 2019; 10:63-89.

12. Alibhai SM, Krahn MD, Fleshner NE, Cohen MM, Tomlinson GA, Naglie G. The association between patient age and prostate cancer stage and grade at diagnosis. BJU Int 2004; 94:303-6.

13. Mir MC, Planas J, Raventos CX, de Torres IM, Trilla E, Cecchini $\mathrm{L}$, et al. Is there a relationship between prostate volume and Gleason score? BJU Int 2008; 102:563-5.

14. Çaliskan S, Kaba SL, Koca O, Öztürk MI. Does small prostate predict high grade prostate cancer? J Coll Phys Surg Pak 2017; 27:97-100.

15. Iqbal N, Bhatti AN, Husain S. Role of digital rectal examination and prostate specific antigen in detecting carcinoma prostate. J Coll Phys Surg Pak 2003; 13:340-2.

16. Fang YQ, Zhou XF, Qiu JG, Wen XQ, Wang DJ, Pang J, et al. Serum psa level and Gleason scores of needle biopsy specimens predict the postoperative pathologic grade of prostate cancer. Acta Med 2013; 29:279.

17. Naz S, Ahmad S, Ghafoor F, Butt NS, Akhtar MW. Free and total prostate specific antigen in benign prostate hyperplasia and prostate cancer. J Coll Phys Surg Pak 2004; 14:69-71.

18. Elabbady AA, Khedr MM. Free/total PSA ratio can help in the prediction of high Gleason score prostate cancer in men with total serum prostate specific antigen (PSA) of 3-10 ng/ml. Int Urol Nephrol 2006; 38:553-7.

19. Drobková H, Jurečeková J, Grendár M, Kliment J, Jr., Halašová E, Kliment J. Testosterone as a prospective predictor of pathological Gleason score and pathological stage in prostate cancer. Gen Physiol Biophys 2017; 36:549-56.

20. Morote J, Ramirez C, Gómez E, Planas J, Raventós CX, de Torres IM, et al. The relationship between total and free serum testosterone and the risk of prostate cancer and tumour aggressiveness. BJU Int 2009; 104:486-9. 